\title{
Oculomotor control in children with Special Educational Needs (SEN): the development and piloting of a novel app-based therapeutic intervention
}

\author{
Rosie Clark ${ }^{1}$ (1) $\cdot$ Cathy Williams $^{1}$ (1) $\cdot$ lain D. Gilchrist ${ }^{2}$ (I)
}

Received: 8 February 2021 / Accepted: 2 June 2021 / Published online: 10 June 2021

(c) The Author(s) 2021

\begin{abstract}
Children with Special Educational Needs (SEN) often have impaired eye movement control which can impact on a wide range of everyday activities including in the classroom, socialising, participation in sport or crossing a road. Although some health practitioners offer eye movement therapies these tend not to have been systematically developed or evaluated. We have developed a new app to deliver eye movement training, based on detailed scientific understanding of eye movement control and extensive clinical experience with this patient group. We first investigated the acceptability of the app within several schools and made modifications based on detailed feedback from the children. Next, we conducted a pilot study to test the feasibility of using the app with 12 children (5-17 years old) who had SEN. The children successfully undertook app-based training for $10 \mathrm{~min}$ per day for 4-5 weeks. There was a high level of attendance and no attrition. We found some variation across participants in their ability to play on the app and the level of engagement. This indicates that pre-assessing children for ability and engagement may be important. This encouraging proof of concept study justifies the development of a full study. Given the time and money spent on existing commercially-offered or internet-based non-validated therapies, this would have substantial patient benefit regardless of the outcome: evidence of no effect could reassure parents that this kind of treatment was not needed, whilst evidence of effect suggests this as a useful treatment for children with poor oculomotor control.
\end{abstract}

Keywords Eye movements · Therapeutic intervention · App $\cdot$ Saccades $\cdot$ Fixation $\cdot$ Smooth pursuit

\section{Introduction}

In humans eye movements are a ubiquitous part of visual behaviour [1]. We move the eyes to stabilise the image on the retina; follow moving objects; and to shift the highresolution fovea to objects of interest in our environment. Many children who have additional learning needs or developmental disorders also have impairments in their ability to appropriately generate these eye movements, and evidence of abnormalities in eye movement control have been seen in

Rosie Clark

r.clark@bristol.ac.uk

1 Population Health Sciences, Bristol Medical School, University of Bristol, 1-5 Whiteladies Road, Bristol BS8 1NU, United Kingdom

2 School of Psychological Science, University of Bristol, 12a Priory Road, Bristol BS8 1TU, United Kingdom children with a wide range of disorders including: Autism spectrum disorder; Attention-deficit/hyperactivity disorder; Friedreich's Ataxia and Epilepsy [2, 3]. Abnormalities of eye movement control are also well documented as part of the spectrum of vision problems in cerebral visual impairment (CVI) [4]. Although definitions vary, CVI affects many aspects of vision and is associated with preterm birth and a wide range of neurodevelopmental disorders [5]. Good eye movement control, both accurately moving the eyes and holding the eye still, is important for a wide range of activities such as reading and learning, socialising with friends, crossing a road or catching a ball $[6,7]$. It is very likely then that poor oculomotor control in children impacts on many areas of life: cognitively, socially and physically.

Two eye movements that can be affected are saccades and smooth pursuit. Saccades move the eyes rapidly to shift the line of sight between successive points of stable fixation where the eye is (relatively) still and smooth pursuit allows clear vision of a moving object as the eyes move to keep 
the image of the moving object projected on the fovea [2]. Several aspects of saccadic control can be impaired; saccades can be slow (velocity), slow to initiate (latency) or hypo/ hypermetric (too small or too large in amplitude) and fixation between saccades can be unstable [2]. The main component of smooth pursuit is the smooth tracking movements of the eye which relies on the ability to predict the motion of the stimuli: small catch-up saccades are used in conjunction with the tracking movements to correct for errors [2, 8]. Impairments in smooth pursuit include problems initiating pursuit eye movement, low gain (accuracy), and saccadic intrusions [2]. Saccadic intrusions are saccadic movements that take the line of sight away from the stimuli of interest [2]. Fixation (keeping a stationary object held steadily on the fovea) is also a key component of oculomotor control that can be impaired. During fixation the eye is not completely stable rather there are very small movements of the eye (including microsaccades) and there is evidence that these are necessary to sustain clear vision [2]. Abnormalities of fixation often are characterised by the presence of square wave jerks and ocular flutter, as seen in patients with Freidrich's ataxia [9]. Often when eye movement impairments are present in a particular clinical population, impairments are found in all three of these aspects of oculomotor control, for example oculomotor abnormalities found in CVI patients include defective coordination and initiation of saccades, instability of fixation and absence of functional smooth pursuit [10].

One way in which children's eye movement control might be improved is by training the eye movements via practice. There is some evidence to suggest that training vision in children who have neurodevelopmental and visual disorders could be beneficial. Rehabilitation in CVI in the most severe cases has focused on the patient's fixation and pursuit of a target [10]. Saccade, tracking (pursuit) and fixation exercises are commonly used rehabilitation methods across different patient groups and ages [11, 12]. A scoping review by Williams and colleagues [13] highlighted two studies where training eye movements resulted in improvements $[14,15]$. More recently, there have been other studies showing evidence that saccadic training can positively influence reading fluency and comprehension [16] and oculomotor control in children with Developmental Coordination Disorder (DCD) [17]. Additionally, other researchers have found a specific form of eye movement training known as 'Quiet Eye training' led to significant improvements in fixation and ability to catch a ball in children with DCD [18].

Although this is encouraging support for eye movement training, in many of these studies the key components of oculomotor control (saccades, fixation and smooth pursuit performance) are not always directly measured (or measured at all) and the therapy used is often very broad or non-specific. Several of these training programmes involve multiple different activities over time, for as long as a year [14, 17]. In addition, many of these therapies are repetitive, monotonous and not engaging and interesting for children, which will have an impact on compliance and attrition particularly in younger children.

More broadly, Kato and colleagues [19] demonstrated that using game-like activities in a therapeutic intervention can effectively increase compliance even in young adults. But there is little evidence that commercially available games lead to improvements in vision that transfer to daily life [20], therefore game-like activities for therapy must be developed from scratch to follow therapy requirements and rigorously evaluated [21]. Specifically, our approach was to ensure that the target skill (eye movement control) must form the basis of the core game mechanic [21].

Our aim for this study and future work is to develop a game-like therapy that is: evidence-based, would directly target eye movement control and could be delivered at school, in the clinic or at home. Here we focus on three main components of oculomotor control that are most easily targeted and as discussed have been shown to improve through training: saccades, smooth pursuit and maintaining fixation. Having developed the app, our longer-term goal is to evaluate the effectiveness of the treatment in a randomised control trial, using objective eye tracking to monitor changes to these oculomotor parameters. We define effectiveness of treatment as objective improvements in the three components of oculomotor control discussed, and improvements in vision-related quality of life. This paper focuses on the development process, along with the acceptability and feasibility of daily training on the app.

We present a novel app for an iPad/tablet designed to train children's oculomotor control, developed by our team at the University of Bristol. We first discuss the development of the app and the modification via children's feedback. We then present an exploratory study on the feasibility of the app intervention for children with SEN.

\subsection{Participatory design and development}

The app was designed by the authors based on extensive clinical experience with this patient group and scientific knowledge of eye movement control. The intention was to make an engaging game-like activity where the generation of appropriate eye movements was essential for performance in the game. However, we did not want to monitor the eye movements of the children while they played. Current eye tracking technology remains cumbersome, not completely robust and expensive and we wanted to ensure that children were easily able to play on a tablet at home or school. In addition, the recording videos, which would be a by-product of eye tracking, brings with it a number of significant data privacy and governance issues.

In order to address this central design constraint, the task for the child in the game is to follow an animal with their 
eyes and monitor a small change in the animal. We designed these changes so that it was only possible to detect them if the child was looking at the animal (i.e. maintaining fixation). As a result, successful detection and responding to the change required that the child had good eye movement control.

The artwork for the app was created by Alex Lucas, a local artist who designed two 'background' pictures for two environments (urban and forest) that can be offered on the first screen when children start the game. She also designed a series of 10 animal characters (5 in each environment) that children could choose to play. The software was made for an iPad by software developer Kieren Pitts, with a view to being able to adapt it for any other tablet platforms (Fig. 1).

The instruction for the child is to follow the chosen animal as it moves around and press anywhere on the screen once the animal has made a small movement of its body. Each individual animal makes a different movement, such as moving its ears, winking an eye, or wagging its tail. To detect these small changes requires the child to fixate the animal. As well as training fixation, half of the time the animal moves around the screen in jumps (initiating saccades) and the other half of the time it moves smoothly (initiating smooth pursuit). If the screen is pressed within a relatively short time frame after the animal has, for example, moved its ears, then a burst of stars is shown around the animal with a corresponding noise. In the corner of the screen, the total number of stars then increases so that children can keep track of their progress and are hopefully encouraged by the reward/positive feedback.

The difficulty of the activity is dynamically altered to reflect the child's performance using a staircase method (see [22]), such that as a child's performance improves at the activity the task itself becomes harder, and if they start to struggle it gets easier again. We manipulate activity difficulty by increasing or decreasing the complexity of the background image as shown in Fig. 2. This ensures that the game is accessible to different ages and abilities, whilst still engaging and allowing for improvements. After several correct responses on a full colour background, the level is then completed, and the child receives a bonus 100 stars.

We conducted 2 rounds of pilot testing with primary school children and used their feedback to improve engagement and accessibility of the app. Out of 52 healthy children tested (aged between 5-10 years), only one 6-year-old found it too hard, all the rest played it for at least 5-10 min, which is a plausible therapeutic "dose". In general, the children were very enthusiastic about playing the app and made encouraging comments.

From the first round of piloting we found that a common feedback from children was that the app would be improved by being able to 'unlock' characters as you progressed. In response to this, we modified the app so that only one animal in each 'environment' was unlocked at

\section{$\mathbf{a}$}
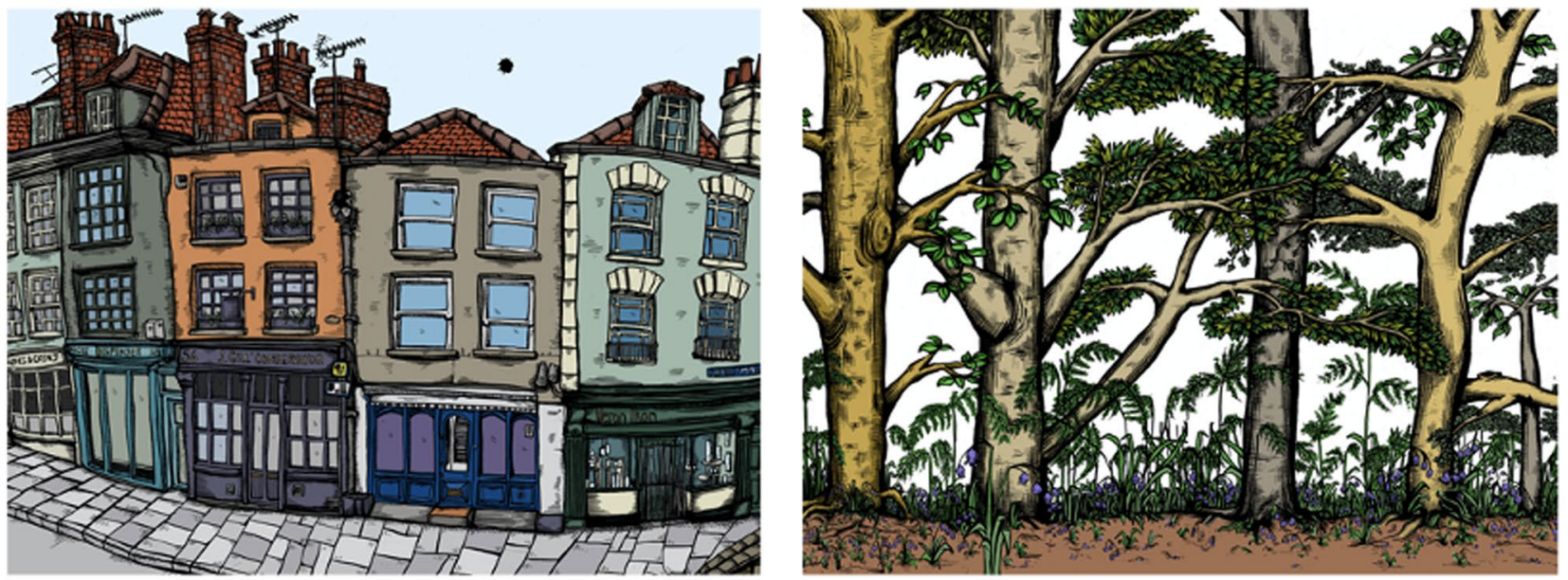

b
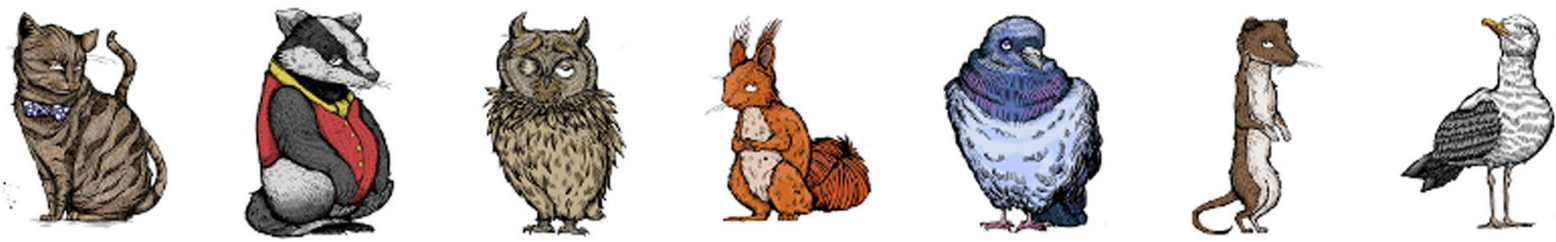

Fig. 1 (a) The urban and forest background for the app, drawn by artist Alex Lucas. (b) Some of the animal characters in the app. The authors and the University of Bristol own the copyright to these images 
Fig. 2 Example of the increase in complexity of the background image in the app; the fox against a section of the 'urban' background scene at each level of complexity

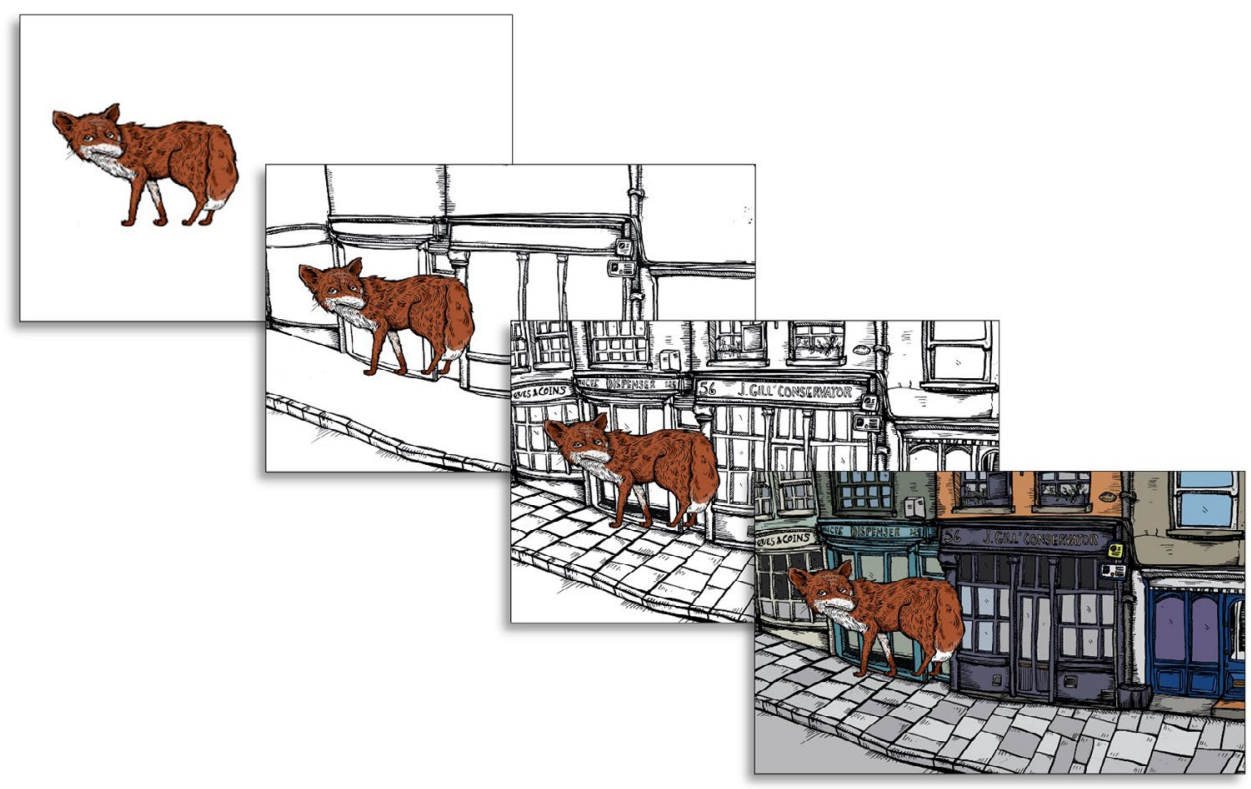

the start. Each time a child completes one round of the activity (from blank screen through to a bonus 100 stars) another animal is unlocked, providing a further rewarding and engaging aspect to the app. Children also identify with this feature as it is a common occurrence in the tablet/iPad activities they had played before.

In the second round of piloting we tested 11 children with SEN (9-17 years). Only one refused to play the activity and 9 out of 11 said when asked that they would play the activity for pleasure. At this stage we also trialled a high contrast version of the activity (shapes rather than animals, on a plain background), designed to ensure the app was as inclusive as possible for children with moderate-to-severe visual acuity impairments or developmental disorders. The task for this version was to press the screen when a letter $\mathrm{T}$ inside the shape flips upside down. The feedback for this was positive, and several children wanted to try this version, so we amended it to be a main option on the home screen rather than accessed only by the researcher. One child who enjoyed this version suggested a more interesting background, so we modified this to a photograph of an aquarium in low contrast black and white, then high contrast and finally full colour (staircase method).

Overall, the piloting led to extensive and successful user driven refinement in the design of the app which we believe made it more accessible and engaging as a therapeutic intervention.

In the next stage in the development of the app we wanted to investigate if it was feasible for children with SEN to engage with the app for the duration and regularity that was likely to be required for it to be used for treatment. We carried out this work in a school setting.

\section{Methods}

\subsection{Participants}

12 children ( 5 female, mean age $=10.3$, range: $5-17$ ) were recruited from two Bristol schools (6 from each) (Table 1). All of the children recruited had SEN and were receiving specialist education. The first school (School A) was a primary school with a resource base for physically disabled and/or visually impaired children. The second school (School B) was a special school for children aged 6-17 with dyslexia and related SEN. At this early exploratory stage, we wanted to be as inclusive as possible and test the feasibility of the therapy for the widest range of children without screening children on specific criteria. The parents of children who the teachers thought might be willing to try the app were invited to take part and given full information about the study and formal consent from parents and assent was given from all children. The study was approved by The Faculty of Science Human Research Ethics Committee at the University of Bristol.

\subsection{Therapeutic procedure}

Each child played on the app for 10 min every day for a period of several weeks, overseen by the lead researcher on the project (lead author). This was scheduled into the school day and took place in a quiet room within each school. Where possible these sessions were timetabled to minimise the impact on the children's school schedule, their SEN support and their wellbeing. These sessions were generally around the same time every day but occasionally had to be moved. As much as possible, the children were encouraged 
Table 1 Details of the diagnoses known to the schools of each child in the exploratory study, showing the heterogeneity of the group

\begin{tabular}{llll}
\hline Participant & School & Age & SEN/Neurodevelopmental disorder \\
\hline 1 & A & 6 & Probable Oculocutaneous Albinism \\
2 & A & 8 & Cerebral Palsy (CP), sensory processing difficulties \\
3 & A & 9 & Hemiplegia, complex medical needs \\
4 & A & 11 & Muscular Dystrophy \\
5 & A & 8 & Born prematurely \\
6 & A & 8 & CP \\
7 & B & 9 & Type 1 Diabetes, Dyslexia \\
8 & B & 8 & Dyslexia \\
9 & B & 17 & Dyslexia, Dyscalculia and Anxiety \\
10 & B & 15 & Limited vocabulary, social skills difficulties, Anxiety, Adopted child \\
11 & B & 11 & Klinefelter syndrome, sensory/OT needs \\
12 & B & 13 & Global Developmental Delay, associated difficulties in language, \\
& & & attention, learning and social skills. Poor gross and fine motor \\
& & & skills \\
\hline
\end{tabular}

to be continually playing the app for the full ten minutes. In School A the length of training was over the course of 5 weeks (23 sessions) and in the second school over 4 weeks (16 sessions) due to a restricted term length in School B.

The app itself collected data about game activity; this included simple information about the time, duration and game-type, and detailed information about each time the screen was pressed and the error rates. Each child had a designated iPad for the study where the data was password protected.

During the study we also trialled two different methods of independently assessing eye movements: objective measurement and clinical assessment. This was to prepare for future studies where children's eye movements will be independently assessed before and after training. Many of the studies discussed in the introduction have used methods of assessing eye movements that are quite rudimentary, subjective and qualitative. It is very possible that eye movement abnormalities and/or changes post-training might be subtle and unable to be detected by such methods. The most effective way to detect and understand these changes would be to use high-resolution eye tracking technology [23]. We trialled this by recording smooth pursuit eye movements using the EyeSeeCam eye tracker (Interaccoustics, Munich, Germany) during the pilot study alongside the use of clinical observations by a specialised Orthoptist. The EyeSeeCam is head-mounted and worn like a pair of swimming goggles, and has a sampling rate of $220 \mathrm{~Hz}$. Eye movements were recorded at a distance of $57 \mathrm{~cm}$ from the screen with a chin rest. Children were calibrated using an in-built calibration programme on the EyeSeeCam software, where they were required to fixate and follow an alien around a screen in a cross shaped formation $\left(15^{\circ}\right.$ in each direction from the centre). The smooth pursuit protocol presented a visual target moving sinusoidally with a period duration of $5 \mathrm{~s}$, each sinusoidal movement (horizontal and vertical) was repeated three times in $15 \mathrm{~s}$. This task was inbuilt into the software for the eye tracker (a closed system) and was child-friendly as they were instructed to follow an alien around rather than a dot or cross. The EyeSeeCam recorded smooth pursuit gain (a measure of accuracy) and velocity. The system provides a pdf. report with these metrics presented, but also saves the raw data for further analysis.

\section{Results}

\subsection{Orthoptist vision assessment}

A vision assessment was carried out in both schools at the start of the study (Table 2). Visual function across the participants was varied.

\subsection{Compliance, engagement and ability}

One child in the study (participant 3 ) missed a high proportion $(52.2 \%)$ of training sessions due to hospital appointments and illness. Therefore we have not included this participant in the results. Of the remaining 11 children, the mean played time on the app per session was 9 min and $51 \mathrm{~s}$ (S.D. $=2.05 \mathrm{~min}$, range $=4.82$ to $16.80 \mathrm{~min}$ ). The mean overall time (across the whole study) played on the app was $2 \mathrm{~h}$ $48 \mathrm{~min}$ (S.D. $=28.53 \mathrm{~min}$, range $=2 \mathrm{~h} 3 \mathrm{~min}$ to $3 \mathrm{~h} 29 \mathrm{~min}$ ). The variability was largely due to the logistical constraints of running the sessions within the school day and factors relating to the children discussed below. The total number of sessions in the first school was 23 and in the second school was 16; sometimes children missed sessions due to illness, appointments and school trips: attendance rates are given in the Table 3. 
Table 2 Orthoptist vision assessment of the participants in the pilot study

\begin{tabular}{|c|c|c|c|c|c|c|c|c|}
\hline School & Participant & Age & $\begin{array}{l}\text { Binocular Visual } \\
\text { Acuity (LogMAR) }\end{array}$ & $\begin{array}{l}\text { Glasses } \\
\text { worn } \\
(\mathrm{Y} / \mathrm{N})\end{array}$ & Test & Cover test result & Nystagmus & AHP \\
\hline A & 1 & 6 & 0.8 & $\mathrm{Y}$ & Keelers Crowded & Moderate left convergent squint & YES & NO \\
\hline A & 2 & 8 & 0.225 & $\mathrm{Y}$ & Keelers Crowded & Alternating divergent squint & NO & NO \\
\hline A & 4 & 11 & 0.0 & $\mathrm{Y}$ & Keelers Crowded & No apparent deviation & NO & NO \\
\hline A & 5 & 8 & 0.8 & Y & Crowded Kays & Moderate right convergent squint & YES & Chin elevation \\
\hline A & 6 & 8 & 0.125 & $\mathrm{Y}$ & Keelers Crowded & $\begin{array}{l}\text { Variable right or alternating con- } \\
\text { vergent squint }\end{array}$ & NO & Head turn (right) \\
\hline B & 7 & 9 & -0.1 & $\mathrm{~N}$ & Keelers Crowded & Small esophoria & NO & NO \\
\hline B & 8 & 8 & 0.1 & $\mathrm{~N}$ & Keelers Crowded & Intermittent left divergent squint & NO & NO \\
\hline B & 9 & 17 & 0.2 & $\mathrm{Y}$ & Keelers Crowded & No apparent deviation & NO & NO \\
\hline B & 10 & 15 & 0.3 & Y & Keelers Crowded & $\begin{array}{l}\text { Small esophoria but right conver- } \\
\text { gent squint without glasses }\end{array}$ & NO & NO \\
\hline B & 11 & 11 & 0.0 & $\mathrm{~N}$ & Keelers Crowded & No apparent deviation & NO & NO \\
\hline B & 12 & 13 & 0.1 & $\mathrm{Y}$ & Keelers Crowded & Moderate right convergent squint & NO & NO \\
\hline
\end{tabular}

AHP Abnormal Head Position

Generally, the participants engaged with the app and expressed enjoyment at the training sessions even after several weeks. However, unlike the experience from the acceptability piloting with typically developing children, there was a broad range of abilities across this heterogeneous group. Table 4 summarises the levels of engagement of the children. Their behaviour was graded using the researcher's prospectively collected session notes. Several of the children struggled with attention and concentration (participants 2, 5 and 12 , categorised as ability rating 1 in Table 4 ), this was often the younger children and/or the children with more complex SEN. Initially these participants were unable to wait for the animal to make a movement and just wanted to press the screen at other times. Pressing the screen at an incorrect time makes the 'trial' restart. Two of these children (participant 5 and 12) did improve slightly and learnt to wait for the

Table 3 Attendance of the participants at the app training sessions, including percentage attendance

\begin{tabular}{llll}
\hline Participant & $\begin{array}{l}\text { Number of sessions } \\
\text { attended }\end{array}$ & $\begin{array}{l}\text { Number of sessions } \\
\text { missed }\end{array}$ & attendance \\
\hline 1 & 19 & 4 & 83 \\
2 & 23 & 0 & 100 \\
4 & 14 & 10 & 58 \\
5 & 22 & 1 & 96 \\
6 & 21 & 2 & 91 \\
7 & 13 & 3 & 81 \\
8 & 16 & 0 & 100 \\
9 & 15 & 1 & 94 \\
10 & 13 & 3 & 81 \\
11 & 16 & 0 & 100 \\
12 & 16 & 0 & 100 \\
\hline
\end{tabular}

animal's movement, but participant 2 struggled with this throughout. The high contrast version of the app activity was very helpful for participant 5 , as they could detect the change in the target shape more clearly. Once they had grasped this version of the activity, they then slightly improved in their ability to play the animal based activity. In order to keep participant 2 engaged in a way that may still train their eye movements, the activity was adapted such that they had to follow the animal and keep pressing the animal itself, while being given targets for the number of presses and counting along themself. The visually guided hand movements will have initiated fixation, saccades and possibly smooth

Table 4 Prospective assessment of the ability and engagement of the children and whether this changed over time

\begin{tabular}{lll}
\hline Participant & $\begin{array}{l}\text { Ability (3=played } \\
\text { well independently; } \\
2=\text { played well with } \\
\text { some supervision; } \\
1=\text { needed constant } \\
\text { supervision and could } \\
\text { not play that well) }\end{array}$ & $\begin{array}{l}\text { Change over time (com- } \\
\text { pletely happy to keep } \\
\text { playing }=3 ; \text { became more } \\
\text { distracted but ok with } \\
\text { encouragement }=2 ; \text { strug- } \\
\text { gled or refused to play } \\
\text { over time }=1)\end{array}$ \\
& 2 & 2 \\
1 & 1 & 2 \\
2 & 3 & 3 \\
4 & 1 & 2 \\
5 & 3 & 2 \\
6 & 3 & 3 \\
7 & 3 & 2 \\
8 & 3 & 2 \\
9 & 3 & 3 \\
10 & 3 & 2 \\
11 & 1 & 2 \\
12 & & \\
\hline
\end{tabular}


pursuit, but they also struggled with poor attention and their hand very quickly becoming tired. In order to compensate for this, the researcher held her hand under theirs sometimes to keep them focused and engaged as a mechanism of joint attention. Although these techniques did help these three participants $(2,5$ and 12) engage in the app, overall their ability was characterised as rating 1 (Table 4) as they still needed constant supervision.

In general, several methods were developed to keep these children focused: frequent encouragement and positive feedback, joint attention (acting as if playing the app 'together'), extra short-term goals (number of presses, correct responses, stars) and even music (the researcher banging a small drum after a correct response).

All the children particularly enjoyed the staggered unlocking of different characters; the more able children (characterised as ability rating 2 or 3 ) found this an attainable and encouraging goal of the activity, whereas the less able children were just excited by the introduction of a new animal. For the less able children animals had to be unlocked before sessions (participants 2, 5 and occasionally participant 1) staggered across the training, as they were very unlikely to be able to get to the stage of unlocking one themselves. We made the decision to do this after training had started and had realised how excited these children were to unlock new animals.

Conversely for the children who were very able to play the activity, we found that some started to lose motivation towards the end of the training as they had unlocked all the animals (participants 6, 8, 9 and 11 categorised as rating 2 for the change over time measure in Table 4). They still seemed to enjoy the app in principle, but they started to be less focussed and would lose concentration as playing the app became less goal directed. For these children we set short-term goals within each session of how many stars they should aim to get. This helped keep them engaged with the task.

All participants undertook their app training sessions with the researcher, apart from participant 9 who had quite severe anxiety around new people. She was the oldest participant and was generally able to play the app independently. After the first session during which her anxiety clearly hindered her ability to focus on the app, the sessions were overseen by an occupational therapist (OT) from the school who frequently worked with her one-on-one. The OT was advised on how best to support the participant during the sessions, and gave frequent feedback on progress. In general the OT reported that the ability to play to app improved over time ("more able to detect the small animal movements"), but that this child did begin to become more distracted towards the end of the training.

\subsection{Trial of eye movement assessment}

Generally objective eye tracking was very challenging in this group. However, the eye-tracking showed an interesting distinction between 'catch-up' and 'intrusive' saccades occurring in smooth pursuit (Fig. 3). Even accurate smooth pursuit will contain small saccades (Fig. 3a), these are not necessarily detrimental and likely serve to compensate for imperfect tracking of the target [2]. However, impaired pursuit also contains larger and more disruptive saccades which we have characterised here as 'intrusive': these take the eye away from the target and impair continuous tracking (Fig. 3b).

The clinical observations carried out were mapped onto a 5-point scale [5] which although assessed by a specialised orthoptist, cannot give the same level of detail as the objective eye tracking as highlighted in Fig. 3. However, unlike objective eye tracking, such a clinical assessment is possible on all children.

\section{Discussion}

Overall, the pilot feasibility study was successful as all of the children continued to attend sessions, engage and play the app for the duration of the study. There was some variation in attendance (mainly due to appointments, school commitments and illness). This is less likely to be a problem if children were able to complete the daily therapy sessions on the app at home, which may be an important method to explore for the next stage of the project.

The study group were very heterogeneous and there was variation in the level of engagement and ability to play the app. We did not pre-assess the participants before conducting the exploratory study, so had very little control over the children's ability, interest and engagement in the app. The results of this study indicates that for future studies on this intervention pre-assessing would be an important component, particularly to ensure that all children have sufficient ability to play the app. Additionally, to ensure the continual engagement on the app we could adapt the app to include more engaging and game-like features (or external rewards such as stickers, toys etc.) to aid the longevity of the more able children's interest: we know that participants must be challenged to generate motivation in therapeutic interventions [21]. These two strategies (selection by assessment and further app development) would reduce the number of participants who were not able to play the app well, increase engagement of the participants who were most able and completed the challenges of the app early on in their training, thus contributing to a more effective therapy. 
a

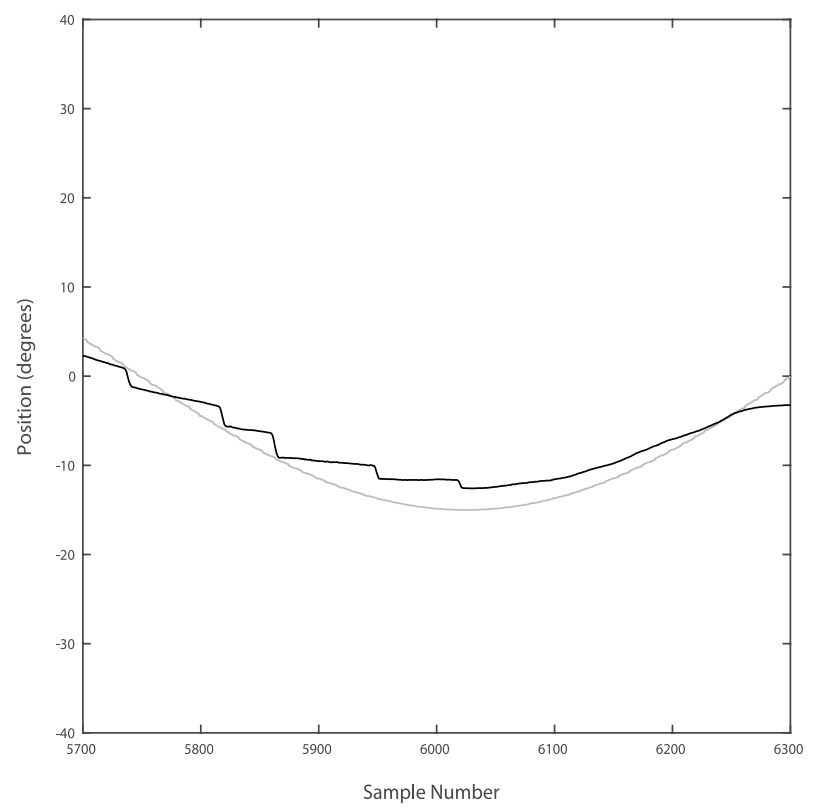

Fig. 3 (a) Graph showing an example eye trace (black line=eye position, grey line $=$ target position) of several catch-up saccades from one participant (b) example eye trace of an intrusive saccade. The smooth

We encountered obstacles trialling eye tracking in this pilot study, however our view is that the challenges we faced with eye tracking these participants could be mitigated by more appropriate technology. The eye tracker we now have available to us would make it possible to monitor oculomotor control in a future study (namely the Portable EyeLink Duo from SR Research Ltd. [www.sr-research.com]). We have used this eye tracker in a clinical setting with children who have a wide range of neurodevelopmental disorders and differing visual function and found it to provide much better quality data. Additionally, it is not a closed system so we have been able to design our own eye movement assessments (similar to the EyeSeeCam assessments) for fixation, smooth pursuit and saccades with engaging animal stimuli and options of matched stimuli for older children. It is also appropriate for use in this population as it only requires the child to wear a small sticker on their forehead, rather than being head mounted. In a future study, using this eye-tracker to objectively measure changes experienced post-intervention with the app will be crucial.

Although we can directly test the oculomotor control of children using clinical observation and/or objective eye tracking, it is unclear how well this translates to their everyday life. Asking a child to fixate a target, or generate a saccade or smooth pursuit while looking at a screen is going to be subject to different constraints than generating this behaviour in the classroom or playground. In future studies, it would be advantageous to measure this in some way, b

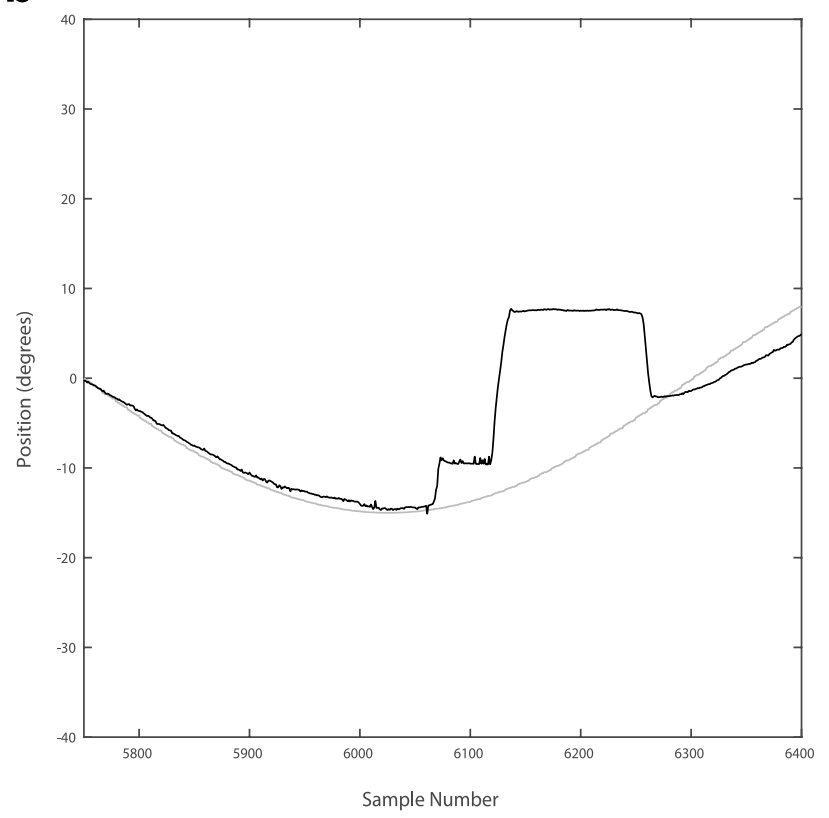

pursuit protocol presents a visual target moving sinusoidally with a period duration of $5 \mathrm{~s}$, each sinusoidal movement is repeated three times in $15 \mathrm{~s}$. The sampling rate of the EyeSeeCam is $220 \mathrm{~Hz}$

for example using table-top search tasks (as described in $[24,25])$ to assess oculomotor control, such as searching for a particular colour $\mathrm{CD}$ on a shelf. Although visual search would primarily be testing saccadic function, it is possible that a similar 'real-world' task could be developed to assess smooth pursuit, such as while watching a film.

The current study has helped clarify what the next steps should be in the development of this project. The first step will be to adapt the app based on what we have learnt from this pilot study. Primarily this would be the inclusion of some "long-term" goals within the game to keep more able children engaged over time. The next step would be a larger study measuring outcomes before and after intervention: objective eye tracking of saccades, pursuits and fixation (using the EyeLink Duo), "real-world" eye movement tasks as discussed, clinical observations and a measure of quality of life. Given our experience from the pilot study, we would also pre-assess participants to ensure: a basic level of ability to engage with the app and some degree of eye movement abnormality across the three aforementioned metrics. Despite this we are aware that there could still be children who struggle to engage over time, but we would devise a guide based on our experience of key strategies to keep children engaged. A recent study has indicated that CVI, including poorly controlled pursuit and saccadic eye movements, is more prevalent than has been appreciated [5] and $42 \%$ of children with SEN had one or more CVI-related vision problem. There is therefore the possibility that many 
children may benefit from the offer of an effective eye movement training app, so further research is needed.

\section{Conclusions}

We have shown that our new app is acceptable and feasible as a treatment with the administration of a daily session played over several weeks by children with SEN. As described, the next steps are to carry out a study including objective eye movement recording before and after treatment and then our aim is to conduct a fully powered RCT to provide robust evidence on the effectiveness of the intervention. Whether this evidence supports the use of eye movement training to improve eye movement control or not, the results will potentially be of substantial benefit to families and children given the costs currently associated with delivering existing untested therapies.

Acknowledgements We thank the two schools we worked with on this project, we are very grateful to them for their support and assistance. We also thank the children who took part at all stages of the pilot study, and their families who supported them. We are incredibly grateful for Alex Lucas' design and artwork for the app, Kieren Pitts for creating and programming the app, and to Penny Warnes for her expert orthoptist vision assessments.

Authors' contributions RC: carried out the pilot study in the two schools, collected and analysed data, drafted the manuscript and contributed to editing and revising the manuscript. $\mathrm{CW}$ : carried out some of the acceptability piloting in schools. IDG and CW: designed the prototype app, supervised and supported the pilot study and contributed to writing and revising the manuscript.

Funding The work was funded by the Elizabeth Blackwell Institute Confidence in Concept grant from the University of Bristol (SSCM RD1977 3320).

Availability of data and material Data available on request, disclosed almost in entirety in manuscript.

\section{Declarations}

Ethics approval The study was approved by The Faculty of Science Human Research Ethics Committee at the University of Bristol.

Consent to participate Parents of children who the teachers thought might be willing to try the app were invited to take part and given full information about the study and formal consent from parents and assent was given from all children.

Consent for publication Parents signed a consent from which stated "I understand that on completion of the study and after giving a summary to the school, the data will be anonymised by removing all links between his/her name and his/her study data. The anonymous data will be made "open" and publicly available."

Conflicts of interest The authors have no conflicts of interest to declare.
Open Access This article is licensed under a Creative Commons Attribution 4.0 International License, which permits use, sharing, adaptation, distribution and reproduction in any medium or format, as long as you give appropriate credit to the original author(s) and the source, provide a link to the Creative Commons licence, and indicate if changes were made. The images or other third party material in this article are included in the article's Creative Commons licence, unless indicated otherwise in a credit line to the material. If material is not included in the article's Creative Commons licence and your intended use is not permitted by statutory regulation or exceeds the permitted use, you will need to obtain permission directly from the copyright holder. To view a copy of this licence, visit http://creativecommons.org/licenses/by/4.0/.

\section{References}

1. Findlay JM, Gilchrist ID. Active vision: The psychology of looking and seeing. No. 37. Oxford University Press; 2003.

2. Leigh RJ, Zee DS. The Neurology of Eye Movements. 5th ed. Oxford University Press; 2015.

3. Sweeney JA, Takarae Y, Macmillan C, Luna B, Minshew NJ. Eye movements in neurodevelopmental disorders. Curr Opin Neurol. 2004;17(1):37-42.

4. Ortibus E, Fazzi E, Dale N. Cerebral visual impairment and clinical assessment: the European perspective. In: Seminars in pediatric neurology. vol. 31. WB Saunders; 2019. p. 15-24.

5. Williams C, Pease A, Warnes P, Harrison S, Pilon F, Hyvarinen L, West S, Self J, Ferris J, CVI Prevalence Study Group, Goodenough T. Cerebral visual impairment-related vision problems in primary school children: a cross-sectional survey. Dev Med Child Neurol. 2021.

6. Foulsham T. Eye movements and their functions in everyday tasks. Eye. 2015;29(2):196.

7. Land M. Eye movements in daily life. In: Chalupa L, Werner J, editors. Vis Neurosci. vol 2. MIT Press; 2004. p. 1357-1368.

8. Luna B, Velanova K, Geier CF. Development of eye-movement control. Brain Cogn. 2008;68(3):293-308.

9. Fahey MC, Cremer PD, Aw ST, Millist L, Todd MJ, White OB, Halmagyi M, Corben LA, Collins V, Churchyard AJ, Tan K. Vestibular, saccadic and fixation abnormalities in genetically confirmed Friedreich ataxia. Brain. 2008;131(4):1035-45.

10. Salati R, Borgatti R, Giammari G, Jacobson L. Oculomotor dysfunction in cerebral visual impairment following perinatal hypoxia. Dev Med Child Neurol. 2002;44(8):542-50.

11. Jafarlou F, Jarollahi F, Ahadi M, Sadeghi-Firoozabadi V. Effects of oculomotor rehabilitation on the cognitive performance of dyslexic children with concurrent eye movement abnormalities. Early Child Dev Care. 2020;22:1-3.

12. Berryman A, Rasavage K, Politzer T, Gerber D. Oculomotor treatment in traumatic brain injury rehabilitation: A randomized controlled pilot trial. Am J Occup Ther. 2020;74(1):1-7.

13. Williams C, Northstone K, Borwick C, Gainsborough M, Roe J, Howard S, Rogers S, Amos J, Woodhouse JM. How to help children with neurodevelopmental and visual problems: a scoping review. Br J Ophthalmol. 2014;98(1):6-12.

14. Sonksen P, Petrie A, Drew K. Promotion of visual development of severely visually impaired babies: evaluation of a developmentally based programme. Dev Med Child Neurol. 1991;33:320-35.

15. Gauthier GM, Hofferer JM. Visual motor rehabilitation in children with cerebral palsy. Int Rehabil Med. 1983;5:118-27.

16. Dodick D, Starling AJ, Wethe J, Pang Y, Messner LV, Smith C, Master CL, Halker-Singh RB, Vargas BB, Bogle JM, Mandrekar $\mathrm{J}$. The effect of in-school saccadic training on reading fluency and 
comprehension in first and second grade students: a randomized controlled trial. J Child Neurol. 2017;32(1):104-11.

17. Coetzee D, Pienaar AE. The effect of visual therapy on the ocular motor control of seven-to eight-year-old children with Developmental Coordination Disorder (DCD). Res Dev Disabil. 2013;34(11):4073-4084.

18. Miles CAL, Wood G, Vine SJ, Vickers JN, Wilson MR. Quiet eye training facilitates visuomotor coordination in children with developmental coordination disorder. Res Dev Disabil. 2015;40:31-41.

19. Kato PM, Cole SW, Bradlyn AS, Pollock BH. A video game improves behavioral outcomes in adolescents and young adults with cancer: a randomized trial. Pediatrics. 2008;122(2):e305-17.

20. Kristjánsson Á. The case for causal influences of action videogame play upon vision and attention. Atten Percept Psychophys. 2013;75(4):667-72.

21. Waddington J, Linehan C, Gerling K, Hicks K, Hodgson TL. Participatory design of therapeutic video games for young people with neurological vision impairment. In: Proceedings of the 33rd Annual ACM Conference on human factors in computing systems. ACM; 2015. p. 3533-3542.
22. Meese TS. Using the standard staircase to measure the point of subjective equality: A guide based on computer simulations. Percept Psychophys. 1995;57(3):267-81.

23. Blundell J, Frisson S, Chakrapani A, Gissen P, Hendriksz C, Vijay $\mathrm{S}$, Olson A. Oculomotor abnormalities in children with NiemannPick type C. Mol Genet Metab. 2018;123(2):159-68.

24. Waddington J, Linehan C, Gerling K, Williams C, Robson L, Ellis R, Hodgson T. Evaluation of Eyelander, a video game designed to engage children and young people with homonymous visual field loss in compensatory training. J Visual Impair Blin. 2018;112(6):717-30.

25. Waddington J, Pickering JS, Hodgson T. The table-top visual search ability test for children and young people: normative response time data from typically developing children. Br J Visual Impair. 2019.

Publisher's Note Springer Nature remains neutral with regard to jurisdictional claims in published maps and institutional affiliations. 